\title{
Selective MMP Inhibition, Using AZD3342, to Reduce Gastrointestinal Toxicity and Enhance Chemoefficacy in a Rat Model
}

\author{
Rachel J. Gibson ${ }^{a, b}$ Ysabella Z.A. van Sebille ${ }^{a, b} \quad$ Hannah R. Wardill ${ }^{b-d}$ \\ Anthony Wignall $^{b}$ Joseph Shirren ${ }^{b} \quad$ Imogen A. Ball ${ }^{b} \quad$ Nicole Williams $^{b}$ \\ Kiara Wanner $^{\mathrm{b}}$ Joanne M. Bowen ${ }^{\mathrm{b}}$ \\ a Division of Health Sciences, University of South Australia, Adelaide, SA, Australia; ${ }^{b}$ Discipline of Physiology, \\ Adelaide Medical School, University of Adelaide, Adelaide, SA, Australia; ${ }^{C}$ Centre for Nutrition and Gastrointestinal \\ Disease, South Australian Health and Medical Research Institute (SAHMRI), Adelaide, SA, Australia; ${ }^{\text {Beatrix }}$ \\ Children's Hospital, University Medical Centre Groningen, Groningen, The Netherlands
}

\section{Keywords}

Adverse drug reactions - Matrix metalloproteinases ·

Gastrointestinal toxicity · Mucositis - Diarrhea ·

Methotrexate · AZD3342 · Intervention · Breast cancer ·

Rat model

\begin{abstract}
Background: The common cytotoxic mechanisms that underpin chemoefficacy and toxicity have hampered efforts to deliver effective supportive care interventions, particularly for gastrointestinal (Gl) toxicity. Matrix metalloproteinases (MMPs) have been implicated in both tumor growth and GI toxicity, and as such MMP inhibitors present as a novel therapeutic avenue to simultaneously enhance treatment efficacy and reduce toxicity. Objectives: The aim of this study was to determine the efficacy of an MMP-9/12 inhibitor, AZD3342, on tumor growth and GI toxicity in a rat model. Methods: Female tumor-bearing Dark Agouti rats $(n=90)$ were divided into 4 groups: vehicle control; methotrexate (MTX); AZD3342, and MTX + AZD3342. Tumors were measured daily (for 5 days) using digital calipers. Gl toxicity was assessed using well-established clinical markers (diarrhea/
\end{abstract}

\begin{tabular}{ll}
\hline KARGER & $\begin{array}{l}\text { ( ) } 2019 \text { The Author(s) } \\
\text { Published by S. Karger AG, Basel Openger }\end{array}$ \\
E-Mail karger@karger.com & This article is licensed under the Creative Commons Attribution- \\
www.karger.com/che & NonCommercial-NoDerivatives 4.0 International License (CC BY- \\
NC-ND) (http://www.karger.com/Services/OpenAccessLicense). \\
Usage and distribution for commercial purposes as well as any dis- \\
tribution of modified material requires written permission.
\end{tabular}

weight loss), histopathological analysis, and functional assessment of intestinal barrier permeability. Results: AZD3342 delayed the onset of severe diarrhea by 1 day (vs. MTX) but was unable to improve the overall severity of diarrhea. No changes were detected in tissue morphology or intestinal barrier function. AZD3342 alone suppressed tumor growth ( $p=0.003$ vs. vehicle) but did not enhance the efficacy of MTX. Conclusions: This study showed partial efficacy of AZD3342 in reducing tumor growth and delaying the onset of severe diarrhea caused by MTX in rats. We suggest further studies be undertaken targeting appropriate scheduling of AZD3342 as well as investigating different cytotoxic therapies that strongly activate MMP signaling.

$$
\begin{aligned}
& \text { (c) } 2019 \text { The Author(s) } \\
& \text { Published by S. Karger AG, Basel }
\end{aligned}
$$

\section{Introduction}

The common cytotoxic mechanisms underpinning both the efficacy and toxicity of chemotherapy have hindered efforts to deliver effective supportive care measures. This is particularly the case for the management 
of chemotherapy-induced gastrointestinal (GI) toxicity, with dysregulated cytotoxicity and inflammation at the core of its pathobiology. GI toxicity affects between 40 and $100 \%$ of patients with cancer treated with chemotherapy [1]. Manifesting as diarrhea, pain, and rectal bleeding, GI toxicity has a significant impact on quality of life, rendering patients housebound due to embarrassment, fatigue, pain, or simply the fear of needing to defecate suddenly. In severe cases, it requires hospitalization and extensive supportive care measures, which can result in chemotherapy dose reductions/delays or complete treatment cessation [2]. In addition, severe GI toxicity predisposes to infectious complications and places patients at a 4-fold higher risk of treatment-related death. The management of these complications requires extensive supportive care measures, increasing the length of hospitalization and contributing to the growing economic burden associated with cancer therapy [3].

Currently, GI toxicity cannot be accurately predicted and is without an effective intervention [2]. As such, the identification of novel therapeutic targets that do not compromise treatment efficacy is critical to improve the management of this significant oncological challenge. Matrix metalloproteinases (MMPs) are a group of zincdependent endopeptidases involved in extracellular matrix homeostasis [4]. We have previously shown that MMPs are upregulated in the gut in a number of preclinical models of chemotherapy-induced GI toxicity $[5,6]$. We have also confirmed these findings in a clinical cohort, with upregulated MMP concentrations in the serum of patients treated with standard dose chemotherapy for breast and colorectal cancer [7]. These findings are of clinical importance as MMP inhibitors have been investigated for their anti-tumor activity, both alone and in combination with chemotherapy $[8,9]$, with data suggesting they may be a valid adjunct to traditional cancer care. Despite this, the effect of MMP inhibition on GI toxicity and tumor kill are yet to be investigated in parallel. As such, paralleled investigation of MMP inhibition on treatment toxicity and efficacy presents as a novel therapeutic approach.

AZD3342 is a 403 D synthetic inhibitor of MMP-8, MMP-9, and MMP-12, with greatest affinity for MMP-9 and MMP-12 [10]. While AZD3342 has demonstrated preclinical efficacy in the repair of colonic injury, it has primarily been studied for its therapeutic potential in chronic obstructive pulmonary disease [10]. For the prevention of preclinical GI toxicity and paralleled impact on chemoefficacy, AZD3342 is an attractive agent due to its narrow spectrum of MMP inhibition, and thus reduced likelihood of adverse toxicity, a common finding in numerous clinical trials using broad-acting MMP inhibitors $[9,11]$. Its affinity for MMP-9 and MMP-12 also holds therapeutic potential as these have both been implicated in the pathobiology of GI toxicity. This is particularly the case for MMP-9, which has been shown to increase in the gut following preclinical irinotecan $[6,12]$, as well as systemically in patients undergoing standard dose chemotherapy [7]. In the case of cancer progression, a number of MMPs have been implicated in cancer progression, invasion, and metastasis, including MMP-2, 9, 11, 12, and $14[13,14]$, with altered expression/activity in the tumor microenvironment, as well as MMP polymorphisms linked with increased susceptibility to various malignancies $[15,16]$. As such, the aim of the current study was to investigate the efficacy of selective MMP inhibition, using AZD3342, on the prevention of GI toxicity while simultaneously assessing tumor burden.

\section{Materials and Methods}

This study is reported using the ARRIVE guidelines for the accurate and reproducible reporting of animal research.

\section{Ethical Statement and Husbandry Specifications}

All animal studies were conducted in accordance with ethics approved by the Animal Ethics Committee at the University of Adelaide and complied with the National Health and Medical Research Council (Australia) Code of Practice for Animal Care in Research and Teaching (2013). Animals were individually housed in conventional, open cages at the Laboratory Animal Sciences Facility of the University of Adelaide. Rats were housed under a 12-h light/dark cycle with ad libitum access to autoclaved standard rodent pellets and sterile water. Sawdust bedding was provided in all cases, as well as crinkle paper and toilet rolls for enrichment. All cages were randomly arranged across racks to prevent potential biases.

\section{Study Design}

GI toxicity and the tumoricidal properties of MMP inhibition were assessed in a tumor-bearing (mammary adenocarcinoma) rat model. Female dark agouti rats weighing 150-170 g (7-9 weeks in age) were used for all experiments. All rats were subcutaneously inoculated with $0.2 \mathrm{~mL}\left(2.0 \times 10^{7}\right.$ cells $\left./ \mathrm{mL}\right)$ mammary adenocarcinoma cells on each flank. Tumors were allowed to form until they reached approximately $1 \%$ of total body weight (approximately 1 week). Rats were then randomly allocated into control or experimental groups: vehicle control (2\% Avicel solution/0.5\% Tween 80, $n=18$ ); AZD3342 (kindly supplied by AstraZeneca, $n=18)$; MTX $(n=18)$, and AZD3342 $+\operatorname{MTX}(n=36)$. Randomization was performed using unblinded randomized number generation in Excel. Minor adjustments were made to ensure experimental groups exhibited comparable baseline body weight and tumor size. 


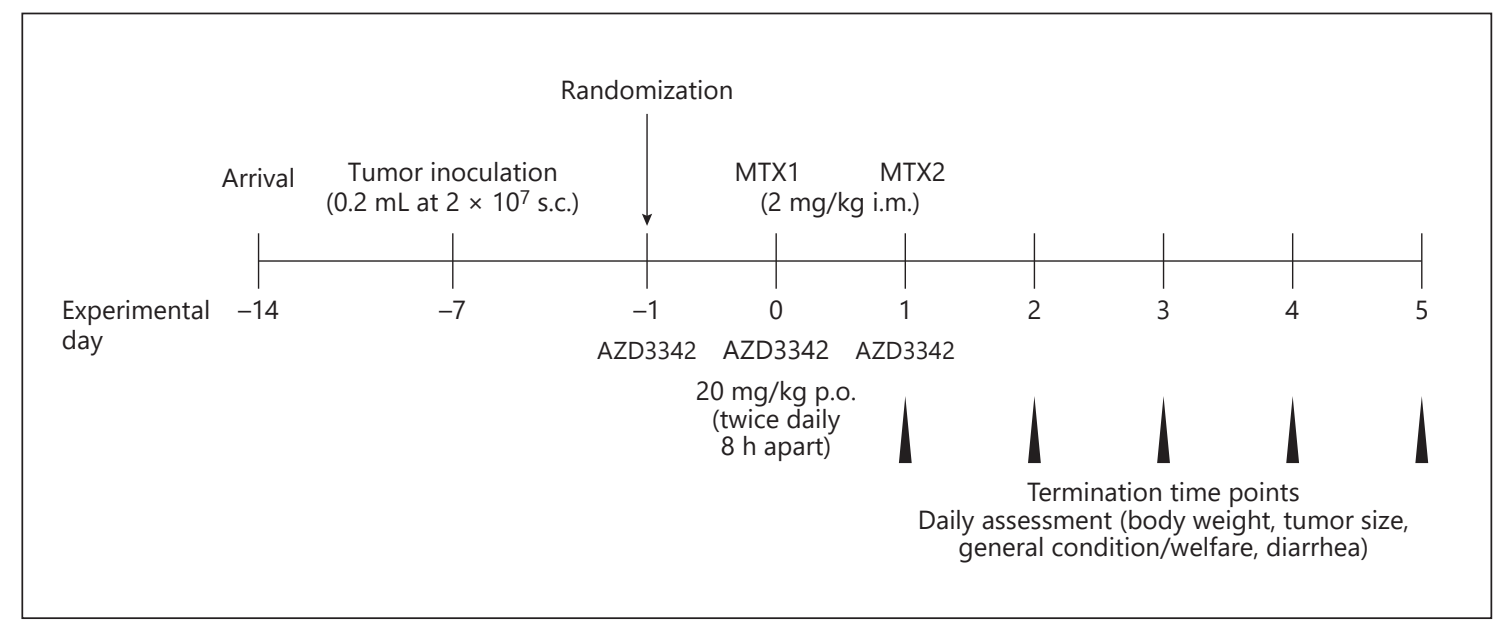

Fig. 1. Experimental timeline. Tumor-bearing rats were randomized to receive vehicle control, AZD3342 alone, MTX + AZD3342 vehicle, or MTZ + AZD3342. MTX was delivered in two intramuscular doses of $2 \mathrm{mg} / \mathrm{kg}$. AZD3342 was administered twice daily $(20 \mathrm{mg} / \mathrm{kg})$ on 3 consecutive days via oral gavage. Rats were euthanized at $24,48,72,96$, and $120 \mathrm{~h}$ after the first MTX injection.

\section{Drugs: MTX and AZD3342}

Methotrexate (MTX, Sigma-Aldrich) was used as a representative chemotherapeutic agent for treatment of breast cancer. The dose of MTX was based on those used in previous cyclic models of GI toxicity in which clinically relevant weight loss and self-limiting diarrhea were observed [17]. AZD3342 (AstraZeneca) is a $403 \mathrm{D}$ synthetic selective MMP inhibitor targeting MMP8,9 , and 12 . Previous reports indicate higher inhibitory capacity for MMP-9 and MMP-12, with $\mathrm{IC}_{50}$ values of $16 \mathrm{nM}$ (MMP-8), $10 \mathrm{nM}$ (MMP-9), and $6 \mathrm{nM}$, (MMP-12) [10]. Previous research has shown that three consecutive subcutaneous doses of $50 \mathrm{mg} /$ $\mathrm{kg}$ is sufficient to increase anastomosis breaking strength and reduce leakage. For maximal contact with the intestinal epithelium, AZD3342 was administered via oral gavage (p.o.) twice daily for 3 days [10]. Due to the targeted luminal exposure and higher frequency of administration, a dose of $20 \mathrm{mg} / \mathrm{kg}$ was selected for all experiments.

\section{Induction of GI Toxicity}

MTX was administered under 3\% isofluorane anesthetic twice (day 0 and day $1 ; 9$ a.m.) at $2 \mathrm{mg} / \mathrm{kg}(6.25 \mathrm{mg} / \mathrm{mL})$ intramuscularly. Control rats received saline injections in an equivalent volume. AZD3342 or vehicle control (2\% Avicel solution/0.5\% Tween $80)$ was administered via oral gavage $(20 \mathrm{mg} / \mathrm{kg}, 4 \mathrm{mg} / \mathrm{mL})$ twice daily ( 9 a.m. and 5 p.m.) beginning on day -1 and ending after the final MTX injection (day 1; Fig. 1). Groups of rats were killed via isoflurane anesthesia, cardiac puncture, and cervical dislocation at 24, 48, 72, 96, and $120 \mathrm{~h}$ after the first dose of MTX.

All procedures were performed in designated procedure rooms within the Laboratory Animal Sciences Facility at the University of Adelaide. The primary outcome of the study was GI toxicity, defined by body weight loss. Secondary outcomes included diarrhea incidence and severity, intestinal injury as determined by histopathology, intestinal barrier function, and tumor burden.

\section{GI Toxicity Assessment}

All rats were monitored twice daily for the presence of diarrhea. Two independent assessors (A.W. and J.S.) quantified diarrhea using a validated grading system where $0=$ no diarrhea, $1=$ mild diarrhea with soft stools and perianal staining, $2=$ moderate diarrhea with loose stools and perianal staining of fur, $3=$ severe diarrhea with watery stools with or without mucous, and fur staining incorporating the hind legs [18]. In addition, rats were weighed daily to track weight loss/gain. Body weight was corrected for tumor weight. Rats were euthanised if they displayed $>15 \%$ weight loss or significant distress and deterioration, in compliance with animal ethical requirements. Assessments were performed at the same time daily (10 a.m. and 3 p.m.) to mitigate potential biases relating to circadian rhythms.

\section{Ex vivo Intestinal Permeability Assessment}

Intestinal permeability was assessed using $4 \mathrm{kDa}$ fluorescein isothiocyanate (FITC)-dextran (Sigma-Aldrich). All rats received a $600 \mathrm{mg} / \mathrm{kg}$ dose $(120 \mathrm{mg} / \mathrm{mL})$ of $4 \mathrm{kDa}$ FITC-dextran via oral gavage $2 \mathrm{~h}$ prior to culling. Blood was then collected via cardiac puncture at necropsy into serum-gel clotting activator tubes and protected from light. Samples were centrifuged at 3,000 rpm for $10 \mathrm{~min}$ and serum isolated. Serum samples were diluted 1:3 with $1 \times$ PBS and quantified using the BioTek Synergy ${ }^{\circledR} \mathrm{Mx}$ Microplate Reader (BioTek, Winooski, VT, USA) and Gen5 version 2.00 .18 software relative to a standard curve (range $0.0001-10 \mu \mathrm{g}$ / $\mathrm{mL})$.

\section{Histopathological Analysis: Tissue Preparation}

At necropsy, the entire GI tract from the pyloric sphincter to rectum was dissected and flushed with chilled isotonic saline to remove intestinal contents. Samples of jejunum and colon were collected and fixed in 10\% formalin for embedding in paraffin. All histopathological analysis was conducted on $5-\mu \mathrm{m}$ sections cut on a rotary microtome and mounted onto glass Superfrost ${ }^{\circledR}$ micro- 
scope slides (Menzel-Gläser, Braunschweig, Germany). Slides were scanned using a NanoZoomer ${ }^{\circledR}$ (Hamamatsu Photonics, Japan) and assessed with NanoZoomer Digital Pathology software view.2 (Histalim, Montpellier, France).

Hematoxylin and Eosin Staining and Analysis

Hematoxylin and eosin staining was performed on the jejunum and colon. A total tissue injury score was generated using a validated grading system based on the occurrences of eight histological criteria in the jejunum, and six criteria in the colon $[19,20]$. These criteria were villous fusion and villous atrophy (jejunum only), disruption of brush border and surface enterocytes, crypt loss/architectural disruption, disruption of crypts cells, infiltration of polymorphonuclear cells and lymphocytes, dilation of lymphatics and capillaries, and edema. Each parameter was scored as absent $=0$, present $=1$, severe $=2$ in a blinded fashion.

\section{Tumor Assessment}

Tumors were measured daily using digital calipers. Tumor burden was calculated as tumor length (width) ${ }^{2} \times \Pi / 6$ and expressed relative to body weight (\%).

\section{Statistical Analysis}

All data were assessed for normality using the D'AgostinoPearson normality test in GraphPad Prism (v.7). In cases of normality, a two-way analysis of variance (ANOVA) with Tukey's post hoc test was used. Nonparametric data were analyzed using a Kruskal-Wallis test with Dunn's post hoc test. $p<0.05$ was considered significant.

\section{Results}

\section{Baseline Data}

In accordance with the ARRIVE guidelines, baseline characteristics of each experimental group were analyzed at $-24 \mathrm{~h}$, prior to the first AZD3342 administration (Table 1). There were no statistically significant differences in body weight or tumor burden between the groups.

\section{Clinical Assessment of GI Toxicity}

Clinical parameters of intestinal injury include diarrhea, weight loss, and intestinal barrier dysfunction. Control rats (vehicle control and AZD3342 alone) did not display clinical markers of GI injury (data not shown). Rats were assessed twice daily for diarrhea severity, with the highest severity of that day used for statistical analysis and presentation. All rats treated with MTX experienced diarrhea (Fig. 2a, b). Rats that received only MTX had grade 3 diarrhea commencing $48 \mathrm{~h}$ after MTX (Fig. 2a). In contrast, rats treated with MTX + AZD3342 had a delay in severe diarrhea which commenced $72 \mathrm{~h}$ after MTX (Fig. 2b). The incidence and severity of diarrhea was similar from $72 \mathrm{~h}$ onwards across groups treated with MTX and MTX + AZD3342.

MMP Inhibition for Prevention of Chemotoxicity
Table 1. Baseline body weight and tumor burden in each experimental group

\begin{tabular}{lll}
\hline & Body weight, $\mathrm{g}$ & Tumor size, $\mathrm{cm}^{3}$ \\
\hline Vehicle $(n=18)$ & $160.47 \pm 1.78$ & $0.68 \pm 0.08$ \\
AZD3342 $(n=18)$ & $158.57 \pm 2.63$ & $0.63 \pm 0.06$ \\
MTX $(n=18)$ & $157.90 \pm 2.28$ & $0.61 \pm 0.05$ \\
MTX $+\operatorname{ADZ3342~}(n=36)$ & $159.25 \pm 2.05$ & $0.61 \pm 0.03$ \\
\hline
\end{tabular}

MTX caused severe weight loss in all animals. AZD3342 was unable to prevent MTX-induced changes in weight loss (mean \pm SEM: MTX, $-13.01 \pm 2.3 \%$; MTX + AZD, $-12.82 \pm 1.73 \%$; Fig. 2c). Similarly, no significant difference in intestinal barrier dysfunction defined by serum FITC-dextran concentration was identified between MTX and MTX + AZD animals (mean \pm SEM: MTX, $4.6 \pm 0.16 \mu \mathrm{g} / \mathrm{mL}$; MTX + AZD, $2.72 \pm 0.76$ $\mu \mathrm{g} / \mathrm{mL}$; Fig. 2d).

\section{Histopathological Assessment}

MTX injury in both groups and was characterized by severe villous atrophy with stunting and fusion of villi, and crypt disruption in the jejunum (Fig. 3). In the congested lamina propria there was an increased inflammatory infiltrate comprised of lymphocytes, macrophages, plasma cells, and neutrophils, and dilatation of lacteals (Fig. 3a). MTX induced small intestinal atrophy, indicated by wet weights measured at necropsy (Fig. 3b). This remained statistically significant until $96 \mathrm{~h}$. Rats treated with MTX had significantly increased injury scores in the jejunum compared to controls at both 48 and $96 \mathrm{~h}(p<0.01$; Fig. 3c). There were no differences in injury score at any time point in the colon (data not shown).

\section{Tumor Burden}

Tumor growth as a percentage of body weight was measured daily. Rats began treatment with either vehicle control or AZD3342 when tumors were approximately $1 \%$ of body weight. Over the time course of the experiment, rats treated with only AZD3342 had significantly smaller tumors at $72 \mathrm{~h}$ (mean \pm SEM: $5.5 \pm 0.40 \%, p=$ $0.003)$ than vehicle controls $(7.4 \pm 0.52 \%$; Fig. $4 a)$. This effect was not maintained at necropsy, with only rats treated with MTX or MTX + AZD3342 showing a significant reduction in tumor weight from $72 \mathrm{~h}$ onwards (Fig. 4b). 

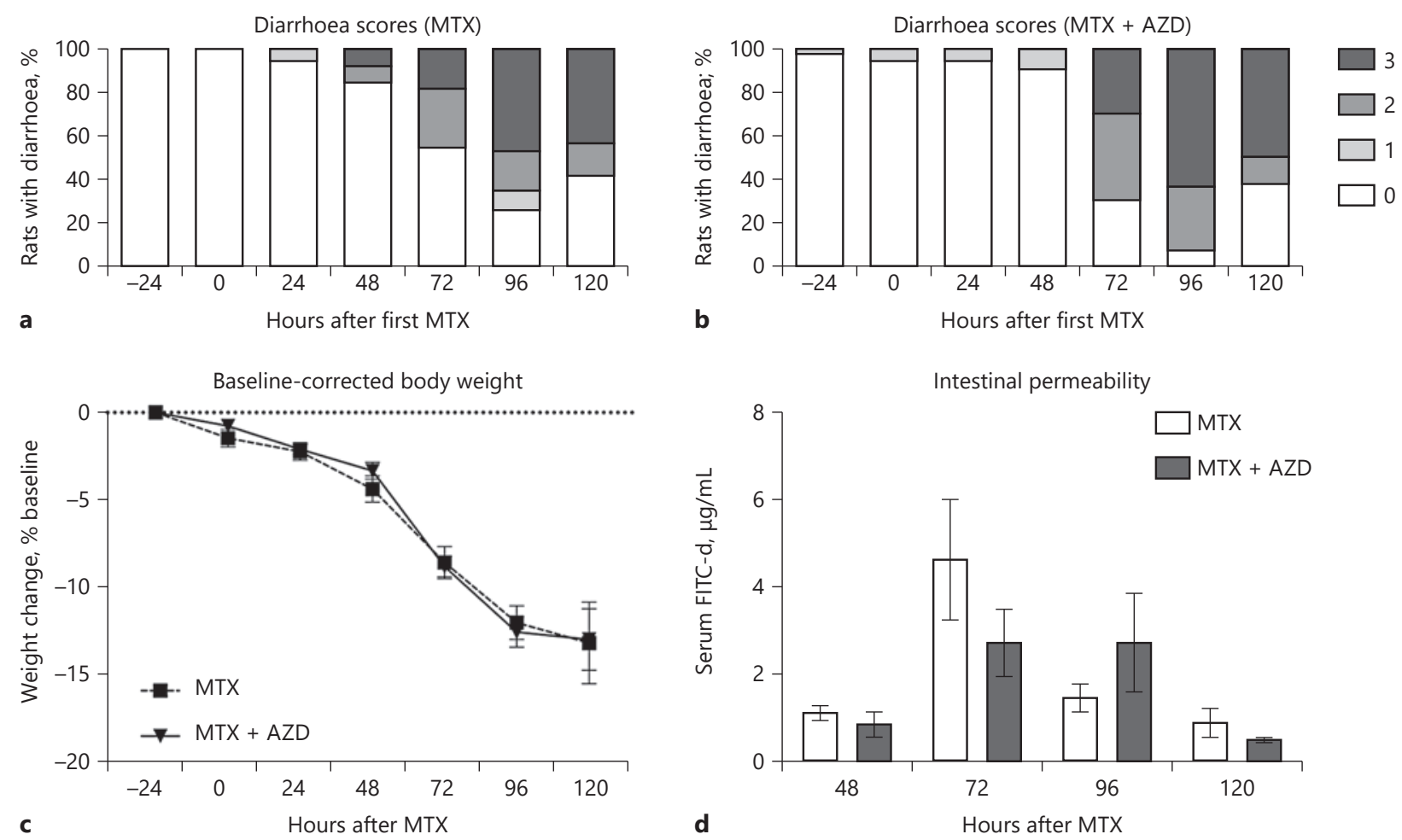

Fig. 2. Clinical markers of GI toxicity. a Rats treated with MTX developed severe diarrhea from $48 \mathrm{~h}$. b Severe diarrhea was not seen until $72 \mathrm{~h}$ in rats treated with MTX + AZD3342. AZD3342 did not reduce the severity of MTX-induced diarrhea from $72 \mathrm{~h}$. AZD3342 did not prevent MTX-induced weight loss (c) or intes- tinal barrier dysfunction (d). Diarrhea data are shown as percentage of animals experiencing grade $0-3$ diarrhea. Weight loss and serum FITC-dextran data are shown as the mean \pm SEM and were analyzed using a two-way ANOVA with Tukey's post hoc test.

\section{Discussion}

There is currently an unmet need to effectively manage GI toxicity without impacting chemoefficacy. MMPs present a promising therapeutic target due to their emerging role in both tumor development and GI inflammation, both of which stem from their regulation and destruction of the extracellular matrix. The current study therefore investigated the potential dual action of an MMP inhibitor, AZD3342, in reducing tumor burden and GI toxicity caused by MTX.

Despite a growing body of preclinical and clinical research implicating MMPs in the development of GI injury, the current study only found modest benefits of AZD3342 in delaying severe diarrhea. AZD3342 did not protect against MTX-induced intestinal barrier dysfunction, weight loss, or histopathological injury. These findings are consistent with previous research showing MMP inhibition with doxycycline was unable to attenuate chemotherapy-induced oral mucositis [21]. They are, however, inconsistent with a growing body of evidence implicating MMPs in intestinal inflammation and demonstrated efficacy of MMP inhibition. Our study also indicated no clinically relevant impact on tumor development when MTX was administered in combination with AZD3342. Although AZD3342 alone reduced tumor size at $72 \mathrm{~h}$, this protection did not translate to other time points and was not observed in the presence of MTX. This highlights the inability of this compound, in this particular preclinical model, to adequately impact on tumor pro- 


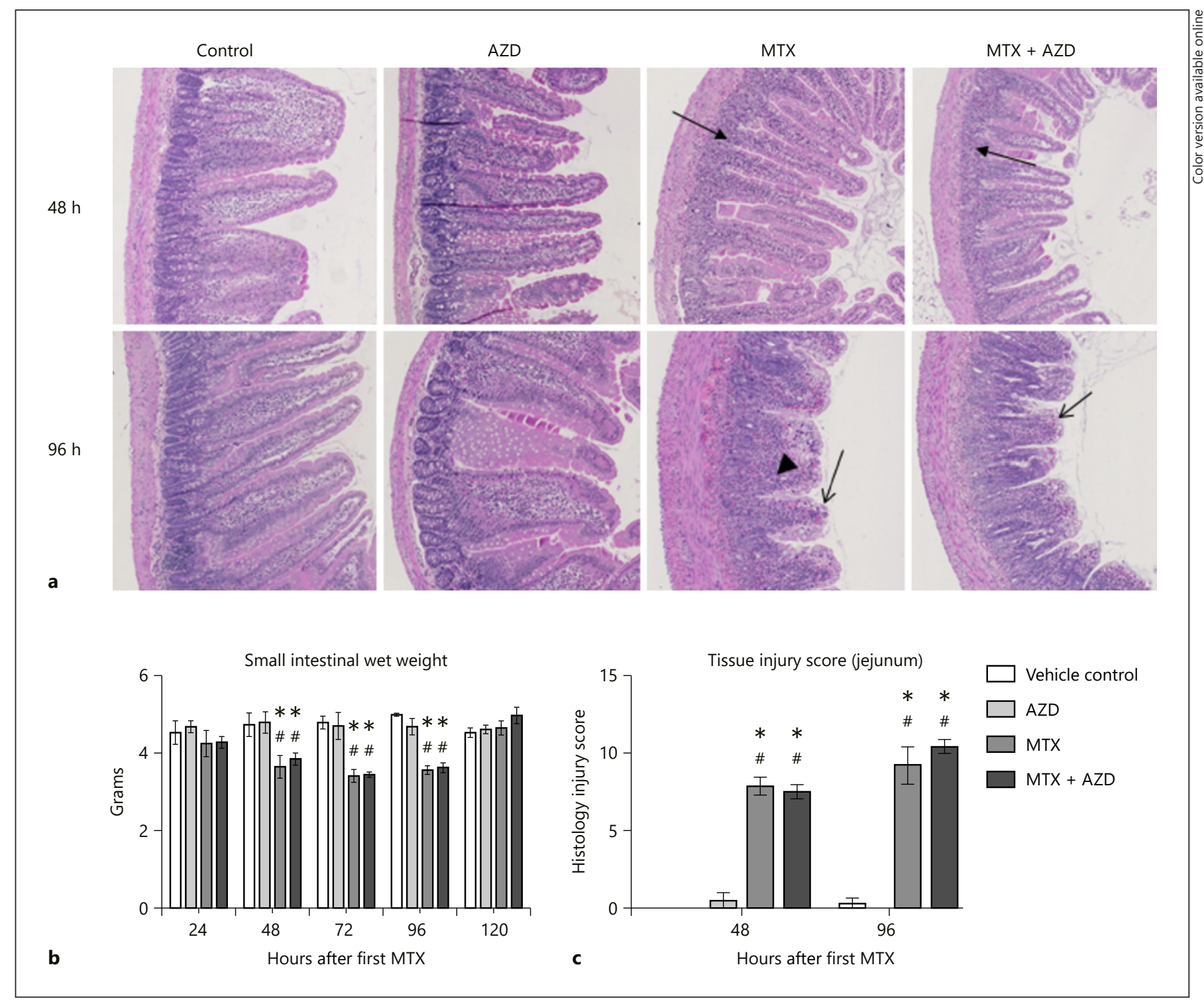

Fig. 3. Intestinal injury assessment. a Representative photomicrographs of the jejunum (original magnification, $\times 100$ ). Arrows indicate crypt loss (filled arrows), surface enterocyte loss and villous blunting (open arrows), and inflammatory infiltrate (arrowhead). b Small intestinal wet weights indicate MTX-induced atrophy. AZ3342 did not prevent MTX-induced small intestinal atrophy. c Histological damage scoring in the jejunum shows a significant increase in injury in groups treated with MTX. AZD3342 did not prevent MTX-induced injury $(p=n s)$. No changes were seen in tissue injury scores for the colon (data not shown). Data are shown as the mean \pm SEM; ${ }^{*} p<0.05$ versus vehicle control, ${ }^{\#} p<0.05$ versus AZD3342 control. All data were analyzed using a two-way ANOVA with Tukey's post hoc test. gression when used in isolation and as an adjunct to chemotherapy.

Clinically, there are conflicting data regarding the efficacy and safety of adjunctive MMP inhibition in cancer treatment. For example, Heath et al. [8] reported reduced tumor growth and spread in patients with a variety of sol- id tumors treated with the MMP inhibitor BAY12-9566 in combination with routine anti-cancer treatment. However, in initial phase I dose-finding studies, significant adverse toxicity was observed, including anemia, shoulder/ back pain, liver abnormalities, nausea, fatigue, diarrhea, rash, and deep vein thrombosis, although none exceeded 


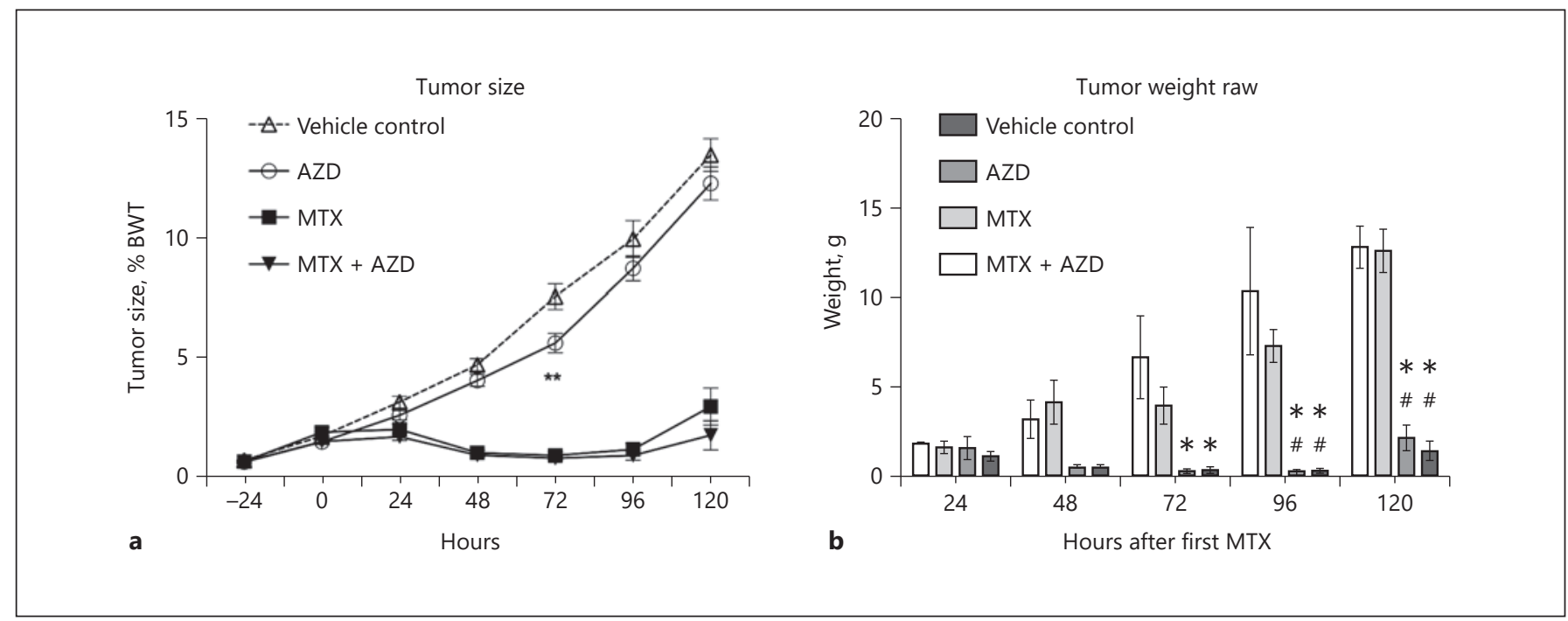

Fig. 4. Tumor growth over 120 h. a MTX and MTX + AZD3342 inhibited tumor size compared to vehicle control and AZD3342 alone. A significant reduction in tumor size was seen at $72 \mathrm{~h}$ between AZD3342 alone and vehicle control (** $p=0.003$ ). b Reduced tumor weight was seen in rats treated with MTX and MTX + AZD3342 compared to vehicle control but no differences between MTX and MTX + AZD3342 were found. This effect was seen at $72 \mathrm{~h}$ (MTX vs. vehicle, ${ }^{*} p=0.001 ; \mathrm{MTX}+\mathrm{AZD} 3342$ vs. vehicle, ${ }^{*} p=0.0003$ ), $96 \mathrm{~h}$ (MTX vs. vehicle, ${ }^{*} p<0.0001$; MTX vs. AZD3342, ${ }^{*} p=0.0002 ;$ MTX + AZD3342 vs. vehicle, ${ }^{*} p<0.0001$; MTX + AZD3342 vs. AZD3342, ${ }^{*} p<0.0001$ ), and $120 \mathrm{~h}$ (MTX vs. vehicle, ${ }^{*} p<0.0001$; MTX vs. AZD3342, ${ }^{\#} p<0.0001$; MTX + AZD3342 vs. vehicle, ${ }^{*} p<0.0001 ;$ MTX + AZD3342 vs. AZD3342, $\left.{ }^{\#} p<0.0001\right)$. All data were analyzed using a two-way ANOVA with Tukey's post hoc test. Data are presented as the mean \pm SEM. grade III or were considered dose limiting. These findings are in contrast to those of Leighl et al. [9] reporting more frequent and severe adverse events (rash, hypersensitivity reactions, and febrile neutropenia) and no improvements in overall survival in patients with advanced non-small cell lung cancer co-treated with the broad-spectrum MMP inhibitor, BMS-275291. It has been speculated that toxicities associated with adjunctive MMP inhibition likely result from specific drug-drug interactions and dysregulated balance between MMP and their natural inhibitory partners, TIMPs (tissue inhibitors of metalloproteinases). This parallels concerns raised in another study using the same broad-acting inhibitor in patients with breast and prostate cancer. In this small phase II trial, increased rates of musculoskeletal toxicity were seen in the experimental arm of the study, with these patients also more susceptible to grade 3 rash and dose-reductions/cessation. These reports highlight the need to pursue MMP inhibition as an adjunct to traditional cancer therapy with extreme caution, with more detailed preclinical investigation.

Given the lack of overwhelming benefit observed clinically for MMP inhibition, the results of the current preclinical study remain difficult to interpret. They are in stark contrast to what has been demonstrated in previous preclinical research, however appear to reflect the lack of efficacy seen clinically. In any case, the results of our study need to be considered within the landscape of our particular preclinical model. Firstly, the dosing schedule chosen for AZD3342 may not have been most beneficial. Given that our previously published data have shown some MMP subtypes maximally increase at $48 \mathrm{~h}$ following cytotoxic therapy [12], further investigations into AZD3342 should test extended therapy protocols that incorporate exposure both before MTX and continuously after MTX to provide maximum coverage. Secondly, the profile of MTX-induced MMP upregulation is not as well characterized compared to that of other chemotherapeutic agents, specifically irinotecan $[6,12]$. While MTX was an attractive agent to use in our model due to its mammary adenocarcinoma target, further investigation of AZD3342 is warranted in preclinical models using other different cytotoxic therapies that strongly activate MMP signaling. MTX dosing schedules may also need to be revised to induce a less severe diarrhea phenotype. It is possible that the severity of mucosal injury observed in this study was so profound that it may have masked any potential benefits of AZD3342. Alternatively, the lack of clinical benefit seen in our study may reflect the inability 
of AZD3342 to effectively inhibit MMPs involved in GI toxicity development. Given that we did not directly assess this in our study, the targeted efficacy of AZD3342 in our model remains unclear.

In conclusion, AZD3342 was not effective at reducing overall MTX-induced GI toxicity and had only a modest impact on mammary adenocarcinoma growth in the Dark Agouti rat. However, given the short delay seen in the onset of severe diarrhea and jejunal tissue injury, we suggest further studies be undertaken to assess the efficacy of AZD3342 in preventing GI toxicity using different cytotoxic therapies that strongly activate MMP signaling. In all future experiments, detailed investigation in tumornaïve and tumor-bearing rats should be performed to dissect the potential of MMP inhibition in GI toxicity and to determine the impact of tumor kinetics on this mechanism. This will enable more insightful results to be achieved regarding the origins of adverse toxicities.

\section{Statement of Ethics}

All animal studies were conducted in accordance with ethics approved by the Animal Ethics Committee at the University of Adelaide (M-2016-030) and complied with the National Health and Medical Research Council (Australia) Code of Practice for Animal Care in Research and Teaching (2013).

\section{Disclosure Statement}

R.J.G. and J.M.B. have previously received support to conduct research from Onyx Pharmaceuticals, Puma Biotechnology, Helsinn Healthcare, and Pfizer Phamaceuticals. R.J.G. is an Executive Board member for the Multinational Association of Supportive Care in Cancer. All remaining authors have no conflicts of interest to declare.

\section{Funding Sources}

H.R.W. is the recipient of an NHMRC CJ Martin Biomedical Fellowship (APP114092). Funding for the study was provided by Adelaide Research and Innovation. We would also like to thank AstraZeneca's Open Innovation Program for freely providing AZD3342 and expertise regarding the compound.

\section{Author Contributions}

R.J.G. and J.M.B. conceived the study, sourced funding, oversaw the animal experiments, and wrote the manuscript. Y.Z.A.v.S. and H.R.W. conducted animal experiments, prepared data for publication, and wrote the manuscript. A.W., J.S., I.A.B., N.W., and K.W. conducted animal experiments, collected data, developed figures, and assisted with reviewing manuscript drafts. All authors have contributed appropriately to be listed and have agreed to the publication of the study results.

\section{References}

1 Al-Dasooqi N, Sonis ST, Bowen JM, Bateman E, Blijlevens N, Gibson RJ, et al.; Mucositis Study Group of Multinational Association of Supportive Care in Cancer/International Society of Oral Oncology (MASCC/ISOO). Emerging evidence on the pathobiology of mucositis. Support Care Cancer. 2013 Jul; 21(7):2075-83.

2 Lalla RV, Bowen J, Barasch A, Elting L, Epstein J, Keefe DM, et al.; Mucositis Guidelines Leadership Group of the Multinational Association of Supportive Care in Cancer and International Society of Oral Oncology (MASCC/ISOO). MASCC/ISOO clinical practice guidelines for the management of mucositis secondary to cancer therapy. Cancer. 2014 May;120(10):1453-61.

3 Carlotto A, Hogsett VL, Maiorini EM, Razulis JG, Sonis ST. The economic burden of toxicities associated with cancer treatment: review of the literature and analysis of nausea and vomiting, diarrhoea, oral mucositis and fatigue. Pharmacoeconomics. 2013 Sep;31(9): 753-66.
4 Sengupta N, MacDonald TT. The role of matrix metalloproteinases in stromal/epithelial interactions in the gut. Physiology (Bethesda). 2007 Dec;22(6):401-9.

5 Logan RM, Stringer AM, Bowen JM, Gibson RJ, Sonis ST, Keefe DM. Serum levels of NFkappaB and pro-inflammatory cytokines following administration of mucotoxic drugs. Cancer Biol Ther. 2008 Jul;7(7):1139-45.

6 Al-Dasooqi N, Gibson RJ, Bowen JM, Logan RM, Stringer AM, Keefe DM. Matrix metalloproteinases are possible mediators for the development of alimentary tract mucositis in the dark agouti rat. Exp Biol Med (Maywood). 2010 Oct;235(10):1244-56.

7 Stringer AM, Al-Dasooqi N, Bowen JM, Tan TH, Radzuan M, Logan RM, et al. Biomarkers of chemotherapy-induced diarrhoea: a clinical study of intestinal microbiome alterations, inflammation and circulating matrix metalloproteinases. Support Care Cancer. 2013 Jul; 21(7):1843-52.
8 Heath EI, O’Reilly S, Humphrey R, Sundaresan P, Donehower RC, Sartorius S, et al. Phase I trial of the matrix metalloproteinase inhibitor BAY12-9566 in patients with advanced solid tumors. Cancer Chemother Pharmacol. 2001 Oct;48(4):269-74.

9 Leighl NB, Paz-Ares L, Douillard JY, Peschel C, Arnold A, Depierre A, et al. Randomized phase III study of matrix metalloproteinase inhibitor BMS-275291 in combination with paclitaxel and carboplatin in advanced nonsmall-cell lung cancer: National Cancer Institute of Canada-Clinical Trials Group Study BR.18. J Clin Oncol. 2005 Apr;23(12):2831-9.

10 Krarup PM, Eld M, Jorgensen LN, Hansen MB, Ågren MS. Selective matrix metalloproteinase inhibition increases breaking strength and reduces anastomotic leakage in experimentally obstructed colon. Int J Colorectal Dis. 2017 Sep;32(9): 1277-84.

11 Bissett D, O’Byrne KJ, von Pawel J, Gatzemeier U, Price A, Nicolson M, et al. Phase III study of matrix metalloproteinase inhibitor prinomastat in non-small-cell lung cancer. J Clin Oncol. 2005 Feb;23(4):842-9. 
12 Al-Dasooqi N, Bowen JM, Gibson RJ, Logan RM, Stringer AM, Keefe DM. Irinotecaninduced alterations in intestinal cell kinetics and extracellular matrix component expression in the Dark Agouti rat. Int J Exp Pathol. 2011 Oct;92(5):357-65.

13 Jena MK, Janjanam J. Role of extracellular matrix in breast cancer development: a brief update. F1000 Res. 2018 Mar;7:274.

14 Han F, Zhang S, Zhang L, Hao Q. The overexpression and predictive significance of MMP-12 in esophageal squamous cell carcinoma. Pathol Res Pract. 2017 Dec;213(12): 1519-22.

15 Banday MZ, Sameer AS, Mir AH, Mokhdomi TA, Chowdri NA, Haq E. Matrix metalloproteinase (MMP) -2, -7 and -9 promoter polymorphisms in colorectal cancer in ethnic Kashmiri population - A case-control study and a mini review. Gene. 2016 Sep;589(1): 81-9.
16 Rollin J, Régina S, Vourc'h P, Iochmann S, Bléchet $\mathrm{C}$, Reverdiau $\mathrm{P}$, et al. Influence of MMP-2 and MMP-9 promoter polymorphisms on gene expression and clinical outcome of non-small cell lung cancer. Lung Cancer. 2007 May;56(2):273-80.

17 Bateman E, Bowen J, Stringer A, Mayo B, Plews E, Wignall A, et al. Investigation of effect of nutritional drink on chemotherapyinduced mucosal injury and tumor growth in an established animal model. Nutrients. 2013 Sep;5(10):3948-63.

18 Bowen JM, Mayo BJ, Plews E, Bateman E, Stringer AM, Boyle FM, et al. Development of a rat model of oral small molecule receptor tyrosine kinase inhibitor-induced diarrhea. Cancer Biol Ther. 2012 Nov;13(13):1269-75.
19 Howarth GS, Francis GL, Cool JC, Xu X, Byard RW, Read LC. Milk growth factors enriched from cheese whey ameliorate intestinal damage by methotrexate when administered orally to rats. J Nutr. 1996 Oct;126(10):251930.

20 Wardill HR, Gibson RJ, Van Sebille YZ, Secombe KR, Coller JK, White IA, et al. Irinotecan-Induced Gastrointestinal Dysfunction and Pain Are Mediated by Common TLR4Dependent Mechanisms. Mol Cancer Ther. 2016 Jun;15(6):1376-86.

21 Ramirez-Amador V, Anaya-Saavedra G, Labardini-Mendez J, Ponce de Leon-Rosales S. Double-blind placebo-controlled randomized clinical trial evaluating doxycycline effects on chemotherapy-induced oral mucositis. J Clin Pharm Ther. 2018 Apr;43(2):202208 